\title{
Structure of the heliospheric current sheet from plasma convection in time-dependent heliospheric models
}

\author{
A. Czechowski ${ }^{1}$, M. Strumik ${ }^{1}$, J. Grygorczuk ${ }^{1}$, S. Grzedzielski ${ }^{1}$, R. Ratkiewicz ${ }^{1}$, and K. Scherer ${ }^{2}$ \\ 1 Space Research Centre, Polish Academy of Sciences, Bartycka 18A, 00-716 Warsaw, Poland \\ e-mail: ace@cbk . waw.pl \\ 2 Institut für Theoretische Physik IV, Ruhr Universitat Bochum, 44780 Bochum, Germany \\ e-mail: kls@tp4.rub.de
}

Received 24 October 2009 / Accepted 23 February 2010

ABSTRACT

\begin{abstract}
Context. The heliospheric current sheet is a plasma layer dividing the heliosphere into the regions of different magnetic field polarity. Since it is very thin compared to the size of the system, it is difficult to incorporate into the numerical models of the heliosphere. Because of the solar magnetic field reversals and the diverging and slowing down plasma flow in the outer heliosphere, the heliospheric current sheet is expected to have a complicated structure, with important consequences for transport processes in the heliosheath. Aims. We determine the shape and time evolution of the current sheet in selected time-dependent 3-D models of the heliosphere, assuming that the heliospheric current sheet is a tangential discontinuity convected by the plasma flow.

Methods. We have derived the shape of the heliospheric current sheet at a given time by following the plasma flow lines originating at the neutral line on the source surface surrounding the Sun. The plasma flow was obtained from numerical MHD or gas-dynamical solutions.

Results. The large-scale structure of the magnetic field polarity regions and the heliospheric current sheet in time-dependent asymmetric models of the heliosphere differs from the results obtained in simpler models. In particular, in the forward heliosheath it is characterized by secondary folds in the heliospheric current sheet that are caused by the solar wind latitudinal variation over the solar cycle. We present examples illustrating some cases of interest: a "bent" current sheet, and the HCS structure during the magnetic field reversal at the solar maximum. We also discuss the evolution of the magnetic polarity structure in the region close to the heliopause.
\end{abstract}

Key words. magnetic fields - plasmas - solar wind

\section{Introduction}

The heliospheric current sheet (HCS) is a narrow plasma layer that divides the heliosphere into the regions with different (open) magnetic field polarity. In interplanetary magnetic field observations it appears as a boundary between the magnetic sectors. The observations made during the crossings of the HCS by the spacecraft (Winterhalter et al. 1994; Zhou et al. 2005) show that it is very thin (of the order of $10^{3}-10^{4} \mathrm{~km}$ within few AU from the Sun) and that the magnetic field does not pass through zero but instead rotates inside the HCS (Smith 2001, 2008). There are also observations of magnetic reconnection at the HCS (Gosling et al. 2006). The large-scale picture of the current sheet emerging from the observations is incomplete, but the results are consistent with the idea that the current sheet can be traced back to the tilted "neutral line" at the source surface surrounding (and corotating with) the Sun and that the current sheet shape is determined by plasma convection (see the reviews by Smith 2001, 2008).

Near solar maximum the HCS extends to high ecliptic latitudes, as confirmed by Ulysses data (Balogh \& Smith 2001; Smith et al. 2001). In this case the very simple picture of the neutral line tilted almost perpendicular to solar equator was found useful to interpret the results (Smith et al. 2001), although more complicated forms of the neutral line are suggested by the observations of the magnetic field on the solar surface.
In the distant heliosphere, the observations of the HCS are due to Voyagers. The sector structure at long distances is more difficult to observe but there is no evidence of fundamental change, like tearing or merging, in the HCS (Smith 2001). When observed, the sector structure was found to be less regular than at shorter distances from the Sun (Burlaga et al. 2003), probably due to interaction of different solar wind streams.

After crossing of the termination shock, the Voyagers continue observing the sector structure (Burlaga et al. 2006, 2007, 2009), implying that the HCS extends into the inner heliosheath. With the distance from the termination shock increasing and the distance to the heliopause going down, the plasma flow deviates more and more from the radial flow (Richardson et al. 2009). This must have consequences for the shape of the HCS and for the magnetic field structure.

In a pioneering work, Nerney et al. $(1991,1993,1995)$ derived a model of the global structure of the magnetic field in the heliosphere (including the inner heliosheath) based on MHD theory and a simple analytical expression for the heliospheric plasma flow. According to their model, the magnetic field forms alternating unipolar and mixed polarity field sectors corresponding to past solar minimum and solar maximum periods. The mixed polarity regions include the heliospheric current sheet. These sectors take the form of "shells" which surround the Sun and expand due to convection by the heliospheric plasma flow. In the inner heliosheath these shells are pressed together due to 
slowing down of the outward flow. In result, the thickness of the magnetic polarity regions decreases towards the heliopause, reaching unrealistically low values and signalling the limit to the model applicability.

Nerney, Suess and Schmahl model is based on the kinematic approximation: that is, the effect of the magnetic field on the plasma flow is not considered. In particular, the axisymmetric stagnation point leads to the unphysical infinity in the magnetic field strength/plasma density ratio at the heliopause (Nerney et al. 1995; Pudovkin \& Semenov 1977a,b). However, the general structure of the plasma flow of their model is also characteristic of all presently studied solutions of the flow dynamics or MHD equations used to model the heliosphere. As long as the magnetic field structure is frozen into the flow, the pile up of alternating mixed and unipolar magnetic polarity regions along the heliopause is a general consequence of the magnetic field reversal at the maximum of the solar cycle.

The structure of the heliospheric magnetic field following from these considerations may be important for many reasons. One is that the tightly folded current sheet near the heliopause is a likely site of magnetic reconnection. Another is the effect of mixed polarity field on the transport of charged particles. As shown by Jokipii \& Levy (1977), drift along the HCS is important for modulation of galactic cosmic rays; the effects of a wavy HCS were also considered (Miyake et al. 2005; Usoskin et al. 2008) Penetration of small charged grains into the heliosphere is facilitated by effective reduction of the magnetic field in the mixed polarity regions (Landgraf 2000; Czechowski \& Mann 2003). Also, the distribution of energetic ( $>28 \mathrm{keV}$ ) particles in the heliosheath, which can now be studied in detail thanks to Voyager observations (Decker et al. 2005, 2008; Stone et al. $2005,2008)$, is likely to be affected by the drift along the folded current sheet extending to high latitudes.

Motivated by these prospective applications, we developed a code which permits effective calculation of the shape of the heliospheric current sheet including the region of the heliosheath and the vicinity of the heliopause. This code can be used with any given model of the heliospheric plasma flow and consequently extends the study of Nerney, Suess and Schmahl to fully 3-dimensional, time-dependent and asymmetric models of the heliosphere.

Similarly to the method of Nerney, Suess and Schmahl, our method is not self-consistent, since the effect of the HCS on the plasma flow is not taken into account. Self consistent solutions require numerical simulations. However, including the HCS in the numerical simulations is very difficult and presently available solutions are not adequate for some applications (see discussion in Sect. 4.2). If our basic assumption that the HCS is approximately a passively convected discontinuity is acceptable, our approach is useful for study of the HCS structures which up to now could not be described by numerical models.

In this work we present some results obtained in this approach concerning the shape of the HCS. We study the effect of time dependence of the flow (on the solar cycle time scale) and of various asymmetries of the heliosphere on the structure of the HCS. The models of the heliosphere used below are different versions of the MHD numerical models derived from the model of Ratkiewicz et al. (1998). We also include two versions of the Bonn model (Fahr et al. 2000), with the magnetic field introduced in the kinematic approximation.

In the vicinity of the heliopause, our results, similarly to those of Nerney, Suess and Schmahl, lead to a pile-up of the magnetic polarity regions originating during the past solar cycles. Clearly, a major rearrangement of the magnetic field and the plasma flow must take place there to avoid unphysical compression of the regions with alternating polarity. Here we assume that it occurs in the immediate vicinity (within some distance $\Delta$ ) of the heliopause, so that the Nerney, Suess and Schmahl picture remains valid in the larger part of the inner heliosheath. Note that many of the features of the heliospheric current sheet described by our model (like secondary folding) extend to the distance of few tens AU from the heliopause, so that the value of $\Delta$ as high as few AU is still consistent with most of our results.

We cannot predict the result of this rearrangement, but we point out two possibilities (Sect. 4.3). One is that the alternating unipolar and mixed-polarity layers near the heliopause will be replaced by a region of disordered field. The global structure of the heliospheric magnetic field is then of the same type as that of Nerney, Suess and Schmahl. The other is that, near the heliospheric stagnation point, reconnection opens a "hole" in the alternating polarity layers and that this "hole" fills with the unipolar field corresponding to the most recent solar minimum. This leads to a picture which, in the forward heliosheath, is similar to the results obtained from some (simplified) numerical MHD calculations.

In Sect. 2, we present briefly our basic assumptions, the models of the heliosphere used in our calculations and the method used to calculate the HCS. In Sect. 3 our results for the HCS structure in the heliosphere, including the inner heliosheath, are presented. In Sect. 4 we compare our results to numerical models and consider possible solutions to the pile up problem. The conclusions are summarized in Sect. 5.

\section{Method}

We assume that the magnetic field in the heliosphere is determined by the boundary conditions, specified at the (spherical) source surface surrounding and corotating with the Sun, and by the freezing-in equations for the given plasma flow outside the source surface. The radius of the source sphere is of the order of few $R_{\odot}$ : for the large-scale structure of the HCS the exact value is unimportant, and we use $R_{\odot}$ in our calculations. The plasma flows are obtained from two different models of the heliosphere:

(A) The MHD model of Ratkiewicz et al. (1998) in two versions: (A1) time-stationary, with isotropic solar wind outflow from the Sun, and (A2) the modified version to incorporate the latitude-dependent (fast and slow) and time-dependent solar wind flow. The model includes the interstellar magnetic field, with strength and orientation chosen to produce a heliosphere with large asymmetry. The same interstellar field configuration was used for an attempt to explain the asymmetry in the termination shock found by Voyagers (Ratkiewicz \& Grygorczuk 2008). The model includes the effects of chargeexchange with the background neutral gas, but in a very crude approximation: the neutral gas density and velocity is taken to be constant over the whole region (Ratkiewicz et al. 2008). The interplanetary magnetic field is also simplified: since the code (which uses a fixed grid) is unable to deal with a thin current sheet, the field is assumed to have the same polarity everywhere inside the heliosphere. This presumes that the presence of HCS (taken to be a passively convected discontinuity) has no strong influence on the flow and that the magnetic pressure distribution is not strongly affected by neglecting the field polarity. The heliospheric current sheet generated by our code is calculated independently of the interplanetary magnetic field following from the MHD model: we use only the velocity field. 
In the MHD model (both A1 and A2 versions) we assume: the interstellar magnetic field strength $3.8 \mu \mathrm{G}$; direction towards $(\lambda, \beta)=\left(68^{\circ},-35^{\circ}\right)(\lambda, \beta$ denote the ecliptic longitude and latitude, respectively); interstellar medium inflow direction towards $\left(74.7^{\circ},-5.2^{\circ}\right)$; neutral hydrogen background density $0.11 \mathrm{~cm}^{-3}$; electron density in the interstellar medium $0.11 \mathrm{~cm}^{-3}$; temperature of the interstellar medium $6400 \mathrm{~K}$. The inner boundary conditions are (density scaled to $1 \mathrm{AU})$ : slow solar wind speed $376 \mathrm{~km} \mathrm{~s}^{-1}$; density $6.36 \mathrm{~cm}^{-3}$; temperature $51109 \mathrm{~K}$; fast solar wind speed $752 \mathrm{~km} \mathrm{~s}^{-1}$; density $3.18 \mathrm{~cm}^{-3}$; temperature $51109 \mathrm{~K}$. In model A1 the solar wind parameters are as for the slow wind.

(B) The Bonn model (Fahr et al. 2000) subsequently developed by Scherer (e.g. Scherer \& Fahr 2003a,b). The model is based on the numerical solution for the axisymmetric flow with 5 components: thermal plasma, pick-up protons, neutral hydrogen, anomalous cosmic rays and galactic cosmic rays. The symmetry axis is the interstellar medium inflow direction. Magnetic fields are not included. The neutral gas is treated as a single fluid, which is known to be a very crude approximation. We use different versions of the model: (B1) time-stationary, and (B2) time-dependent with the solar wind flow speed varying over the solar cycle.

The parameters used in the Bonn model: interstellar medium: neutral hydrogen density $0.2 \mathrm{~cm}^{-3}$; electron density $0.04 \mathrm{~cm}^{-3}$; temperature $8000 \mathrm{~K}$; solar wind: slow wind speed $300 \mathrm{~km} \mathrm{~s}^{-1}$; density (scaled to $1 \mathrm{AU}$ ) $6.25 \mathrm{~cm}^{-3}$; fast wind speed $800 \mathrm{~km} \mathrm{~s}^{-1}$; density $=2.32 \mathrm{~cm}^{-3}$; temperature $2 \times 10^{5} \mathrm{~K}$ (both fast and slow).

These models have a common disadvantage of using a fixed grid. In consequence, they can at best be considered as providing sample flows with the global structure and dimensions approximating the real heliosphere. The limited grid resolution is the reason why some effects of interest, like the HCS evolution in the presence of short time scale variations in the plasma flow velocity, couldn't be included in the present study.

The HCS originates in a (time-dependent) neutral line at the source surface. In most of the examples discussed here, the neutral line is a great circle dividing the source sphere into two halves, one with the outgoing and the other with incoming field lines. The tilt of the neutral line (the angle between the normal to the great circle and the solar rotation axis), is assumed to change linearly with time: $\alpha(t)=\pi t / T$, where $t$ is the time measured from the solar minimum and $T=11$ years is the period of solar activity. The exception is the case illustrated in Fig. 6 where we assumed a non-linear behaviour to expand the low tilt periods: see Fig. 1. For the case of the MHD model A2 we ensure that the tilt of the neutral line is such that the neutral line stays within the slow solar wind region.

Since we concentrate on the large-scale structure of the HCS, this simple model of the neutral line is sufficient for our purpose We have also considered the case with a change in the neutral line topology (a single circle splits into two separate circles on the source surface near solar maximum) based on the observations by the Wilcox Solar Observatory (http://wso. stanford.edu). This case will not be discussed here.

The shape of the HCS at the time $t$ is calculated as follows. We select $M$ points (numbered $i=1 \ldots M$ ) on the neutral line and calculate the flow lines starting from these points at different initial times $t_{j}\left(j=1 \ldots N, t_{j+1}>t_{j}, t_{j}<t\right)$, with the end times fixed to $t$, a common value. The end points, parametrized by $i$ and $j$, approximate the HCS surface; for each $j$, the $i=1 \ldots M$ end points form an image of the neutral line mapped by the flow onto the heliosphere.

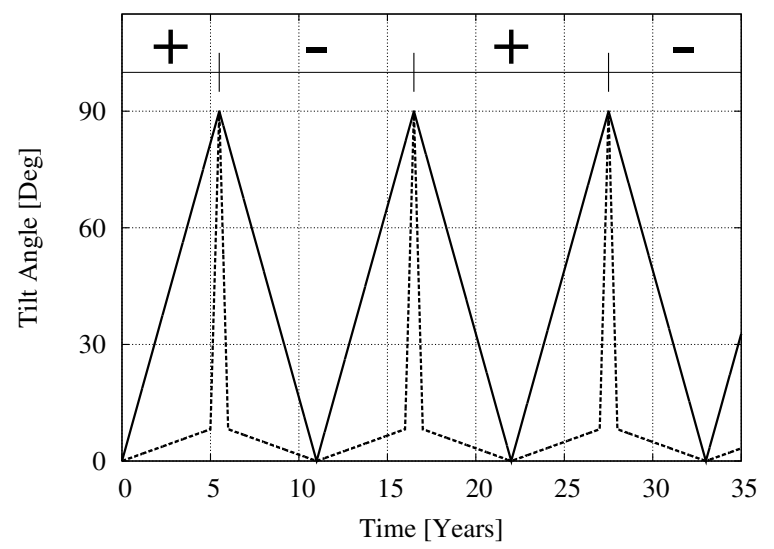

Fig. 1. Tilt angle of the neutral line as a function of time assumed in the calculations. The linear time dependence (solid line) was used in all cases except for the one illustrated in Fig. 6, where the time dependence was changed to nonlinear (dashed line) in order to extend the relative duration of the low tilt periods. +/- signs mark the periods of different magnetic field polarity.

\section{Results}

A brief summary of the structure of the heliospheric flow may help to understand the figures. The solar wind outflow from the Sun is supersonic and approximately radial upstream from the termination shock. The flow slows down and departs from radial in the inner heliosheath, downstream from the termination shock. The heliopause is the boundary between the solar plasma and the plasma of the interstellar medium. Because of the motion of the Sun relative to the interstellar medium, the plasma flow in the heliosheath has a stagnation point structure. The stagnation point (where the plasma velocity is zero) develops at the "nose" of the heliosphere, near the inflow direction of the interstellar medium. The flow turns away from the "nose", towards the heliospheric tail. In consequence, the flow near the nose is diverging, and (towards the stagnation point) slowing down. The compression and stretching of the HCS follows from this flow structure. In the following figures (Figs. 2, 5, 6, 8) the inflow direction is from the left (negative $X$ axis) and the "nose" of the heliosphere is on the left side of the figures.

We present most of our results for the HCS shape in the form of the HCS intersection with the "meridional" plane (we use this name for the plane defined by the Sun position, the solar rotation axis and the interstellar inflow direction). For the MHD model we use the $\left(\boldsymbol{B}_{\infty}, \boldsymbol{V}_{\infty}\right)$ plane (Sun position, interstellar magnetic field $\boldsymbol{B}_{\infty}$ and the interstellar inflow direction $\boldsymbol{V}_{\infty}$ ) which, for the assumed $\boldsymbol{B}_{\infty}$, is close to (18 ${ }^{\circ}$ off) the meridional plane. The Sun is at the origin of coordinates.

The $\left(\boldsymbol{B}_{\infty}, \boldsymbol{V}_{\infty}\right)$ plane is defined by the $\boldsymbol{B}_{\infty}$ direction $(\lambda, \beta)=$ $\left(68^{\circ},-35^{\circ}\right)$ and the $\boldsymbol{V}_{\infty}$ direction $=\left(74.7^{\circ},-5.2^{\circ}\right)$ in ecliptic coordinates. These values are used in the MHD model A2.

\subsection{Solar cycle effect on HCS}

Figures $2 \mathrm{a}-\mathrm{c}$ show the influence of the solar cycle on the structure of the HCS. Moving away from the Sun, the shape of the HCS reflects the past evolution of the tilt of the neutral line.

At solar minimum (case (a)), the tilt of the neutral line is small. The HCS emerging from the Sun is almost flat. At longer distance the HCS oscillation increases in angular amplitude because of higher tilt of the neutral line at the moment of emission. Outside of the HCS envelope there are two unipolar regions, 

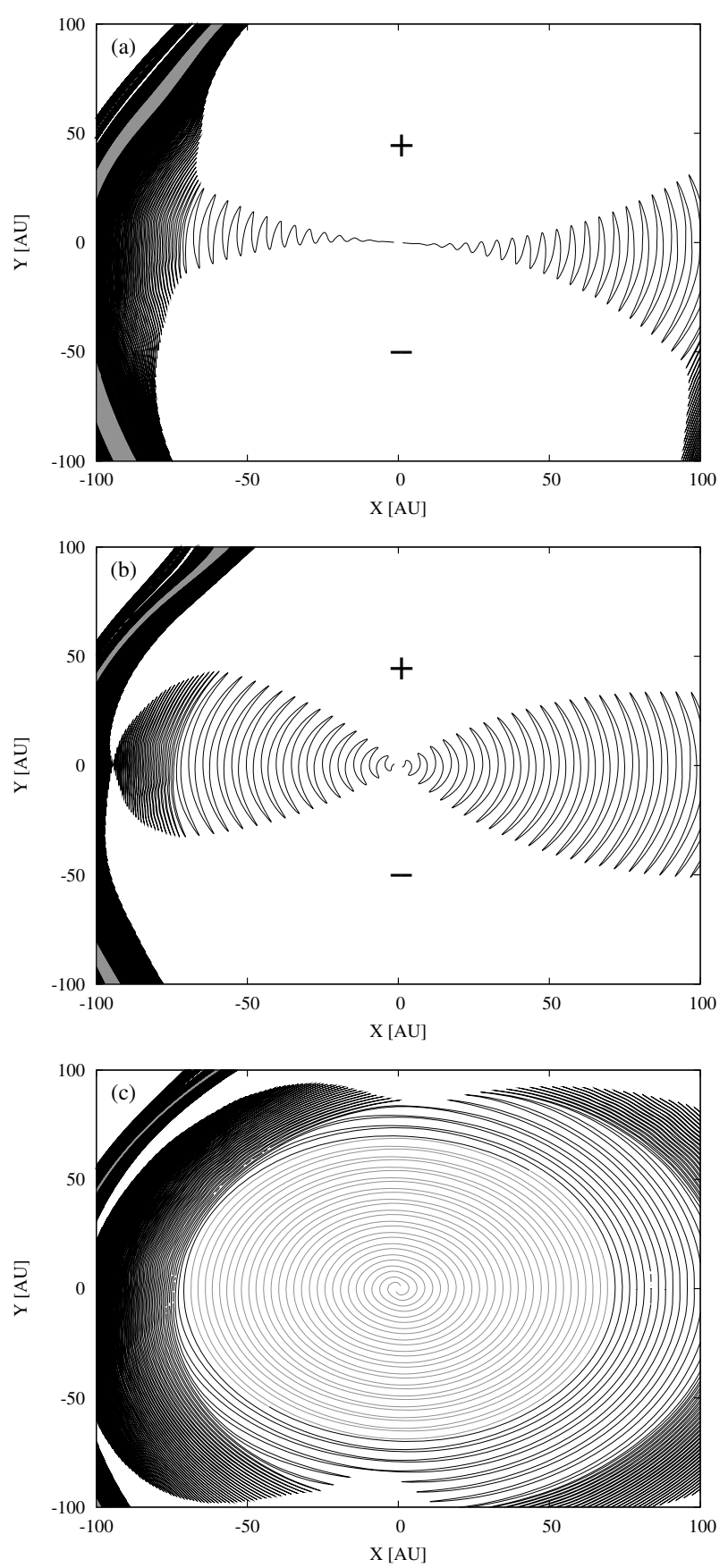

Fig. 2. Intersection of the heliospheric current sheet structure with the $\left(\boldsymbol{B}_{\infty}, \boldsymbol{V}_{\infty}\right)$ plane at a) minimum, b) intermediate phase, and c) maximum of the solar cycle for the MHD model A2. Interstellar inflow direction is horizontal from the left. Sun is at the origin of coordinates. Direction north is approximately down.

each corresponding to a definite field polarity: + for outgoing, for incoming. The region filled by the HCS is not unipolar, since the polarity changes on each crossing of the HCS: the mixedpolarity region. The "wavelength" $(2 \times$ distance between the subsequent folds) of the HCS is given by the local plasma velocity times the solar rotation time. The abrupt decrease of the "wavelength" (tightening of the folds) marks the position of the termination shock. Beyond the termination shock the plasma velocity continues to decrease and the HCS becomes more tightly folded. The mixed polarity region filled with HCS expands in latitude because of diverging plasma flow and further increase

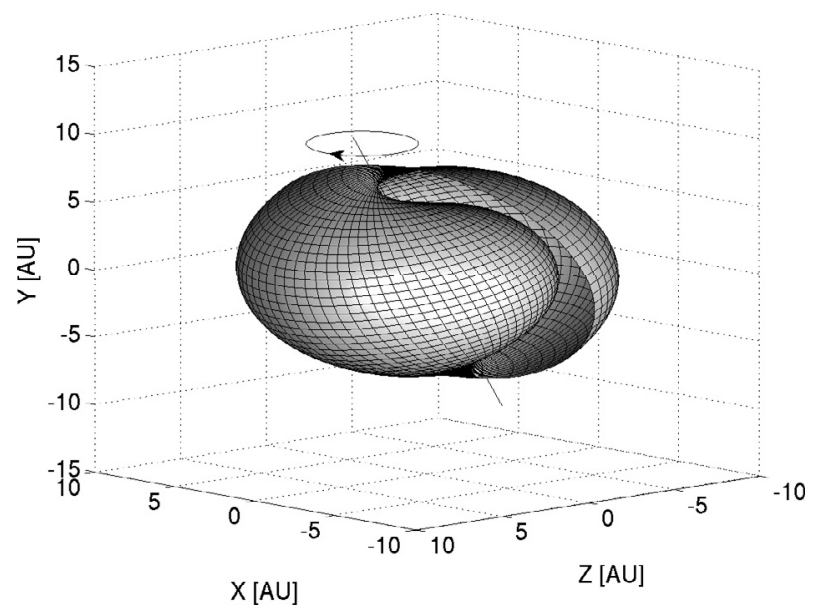

Fig. 3. The heliospheric current sheet in model A1 near the Sun at solar maximum. The Sun is at the origin of coordinates, inside the shell.

of the tilt. The grey area marks the parts of the HCS emitted within 1 year from the solar maximum.

In the intermediate phase (b), the point of minimum tilt of the HCS moved already beyond the termination shock. Near the Sun the angular amplitude of the HCS is now increasing towards the Sun because of the approach of the next solar maximum. The mixed polarity region seen in case (a) is now compressed (the region to the left of the minimum tilt point): a new mixedpolarity region is starting to build up.

At solar maximum (case (c)), the HCS near the Sun extends to all latitudes. New unipolar regions will appear after the maximum, with reversed polarity.

For completeness, we show here also the evolution of the HCS structure near the Sun following from our model during field reversal. Near the solar maximum, the HCS in the vicinity of the Sun takes the form of a spiral "snail shell" shown in Fig. 3 for the moment of time $t$ immediately after the reversal. In our model the HCS consists of the plasma packets emerging from the neutral line on the source surface and moving outward with the plasma flow. Each of the "meridional" lines at the surface of the shell links the points that emerged from the neutral line at the same moment of time; the "parallel" lines link the points that started from the same point of the neutral line at different times.

Figure 4 shows the intersection of the HCS with the meridional plane (a) and with the $\left(\boldsymbol{B}_{\infty}, \boldsymbol{V}_{\infty}\right)$ plane (b) within $20 \mathrm{AU}$ from the Sun: the sections of the "snail shell" similar to that shown in Fig. 3. The dotted line marks the plasma flow lines emerging from the solar poles. At the moment of field reversal the neutral line passes through the solar poles. In consequence, the HCS surface passes through the flow lines emerging from the solar poles. At the moment of time to which the figure corresponds (about a month after the magnetic field reversal) this part of the HCS moved already to the distance of about $10 \mathrm{AU}$ from the Sun. This can be seen (shown by the bold line) in the intersection with the "meridional" plane which contains the solar rotation axis (Fig. 4a, bold lines) but not with the $\left(\boldsymbol{B}_{\infty}, \boldsymbol{V}_{\infty}\right)$ plane (Fig. 4b) because the latter is too distant from the solar rotation axis.

Our model of the solar wind flow and the neutral line evolution (great circle in uniform rotation) is meant to describe the large-scale HCS structure. It is too simple for a realistic description of the HCS structure near the Sun. For calculations with this aim, using the neutral line and the solar wind evolution based 

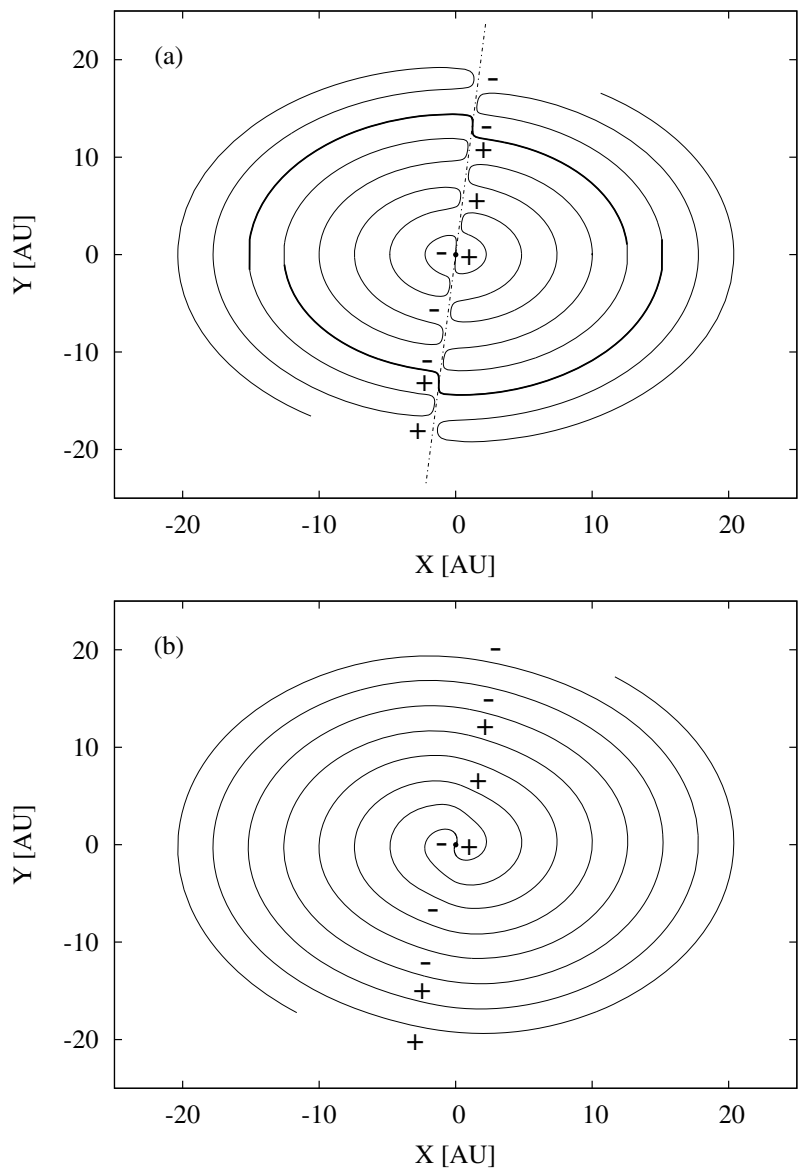

Fig. 4. Intersection of the heliospheric current sheet in model A1 (shortly after solar maximum) with a) the meridional plane and b) the $\left(\boldsymbol{B}_{\infty}, \boldsymbol{V}_{\infty}\right)$ plane. North is approximately downwards. Note the apparent abrupt change in the HCS structure (bold lines) when the HCS surface passes through the solar rotation axis. The $\left(\boldsymbol{B}_{\infty}, \boldsymbol{V}_{\infty}\right)$ plane $\left.(\mathbf{b})\right)$ is tilted too far from the solar axis for this passage to be visible.

on observations but restricted to within $5 \mathrm{AU}$ from the Sun, see Riley et al. (2002).

\subsection{Global structure}

The plasma flow lines emerging from the solar equator form a surface ("solar equatorial surface") that divides the heliosphere into two parts ("hemispheres") each filled by the plasma coming out of one of the solar hemispheres. The parts of the HCS that emerge from the Sun during the periods close to the solar minima (low tilt of the neutral line) would be close to this dividing surface. However, the field reversals during solar maxima imply that no definite polarity can be assigned to any hemisphere: instead, the unipolar regions of opposite polarity alternate in each hemisphere, and must be separated from each other by appropriate boundary regions (mixed polarity regions), that contain the parts of the current sheet emerging from highly tilted neutral line. The mixed polarity regions form "shells" around the Sun (Figs. 5, 6). Together with unipolar regions, these expand with the plasma flow and (in the forward part of the heliosphere) ultimately approach the heliopause. With the assumption that convection solely determines the field structure, these "shells" cannot intersect and the inner "shell" must stay inside the outer one.

Figure 5 shows the intersection of the HCS (calculated for the MHD model A2) with the $\left(\boldsymbol{B}_{\infty}, \boldsymbol{V}_{\infty}\right)$ plane at solar minimum.

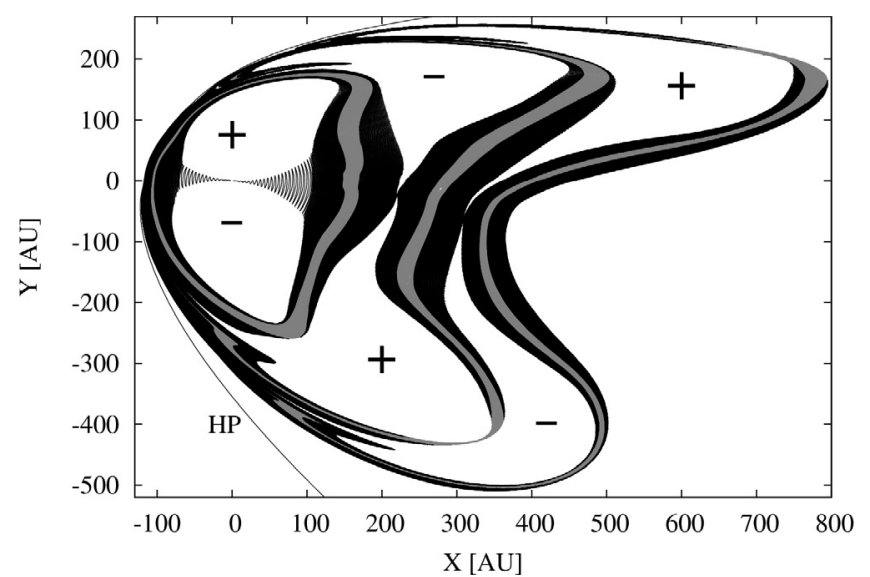

Fig. 5. Intersection of the heliospheric current sheet with the $\left(\boldsymbol{B}_{\infty}, \boldsymbol{V}_{\infty}\right)$ plane at solar minimum for time-dependent MHD model (A2). Plus and minus signs denote the polarity (field lines emerging or incoming relative to the source surface) of the unipolar field regions. The heliopause is marked HP. Interstellar inflow is from the left. Direction to north is approximately downwards.

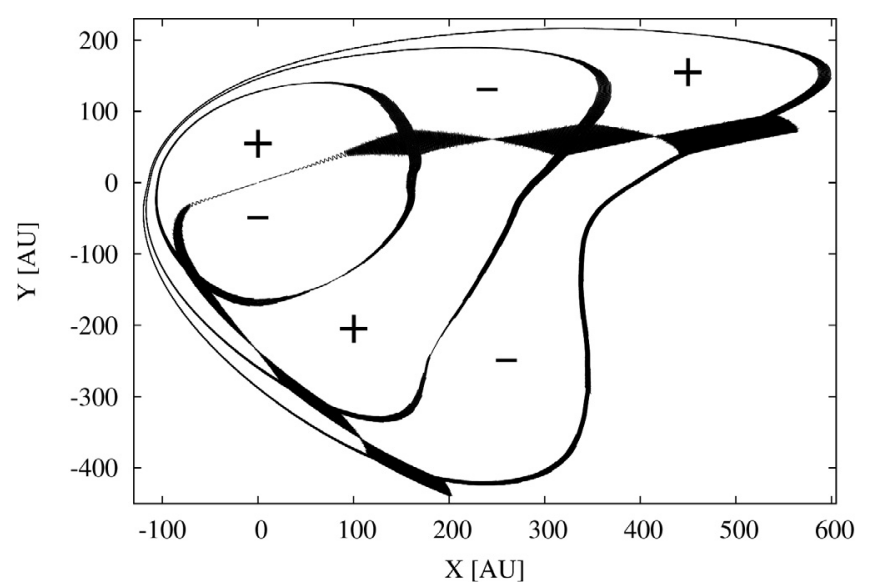

Fig. 6. Intersection of the heliospheric current sheet with the meridional plane at solar minimum for the model with increased angle between the solar equator and the inflow direction of the interstellar matter (see the discussion in the text). The time-stationary MHD model (A1) is used. Interstellar inflow is from the left. Direction to north is approximately downwards.

The wavy current sheet inside the termination shock is clearly visible. Beyond the shock the distance between the subsequent waves becomes too short to distinguish them: the dark areas in the figure are filled by oscillating current sheet (the detailed structure can be seen in Fig. 2a which show part of Fig. 5 in expanded view). Three "rings" represent the expanding mixed polarity regions surrounding the Sun. They originate from three preceding solar maximum periods (high tilt of the neutral line) and are filled by the HCS extending to high heliolatitudes. Printed in grey are the parts of the HCS that originated within 1 year from a solar maximum. Not shown are the parts of the HCS originating earlier than 3.511 -year periods before the illustrated minimum: these parts would appear between the heliopause and the outer ring. The white regions are unipolar. The polarity of the neighbouring unipolar regions alternates.

Another aspect of the HCS global structure is illustrated by Fig. 6. In this case we exaggerated the angle between the interstellar inflow direction and the solar equator plane in order to increase the deflection of the solar equatorial surface and of the 


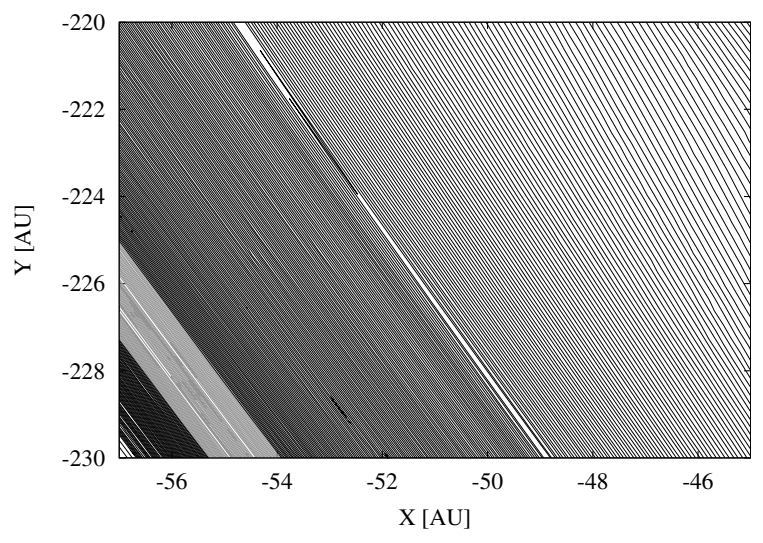

Fig. 7. A fragment of Fig. 5 showing the signature of a bent HCS. The narrow white gap is the unipolar region flanked by two mixed polarity regions filled by the oscillating HCS. For two solar rotations the HCS tilt is low enough to keep it within 1-2 AU from the bent solar equatorial surface. This low tilt segment of the HCS is seen bridging the gap.

HCS. Also, in order to make the HCS deflection more visible, the tilt of the neutral line was assumed to be low for most of the time, except for short periods near the solar maxima. The HCS (the low tilt parts) can be clearly seen to be bent to the north (downward in the figure). The high tilt parts of the current sheet form shells, in this case thinner than in Fig. 5. The plasma flow is derived from model A1 (isotropic solar wind).

In Fig. 5, with a more realistic choice of model parameters (stagnation point close to the solar equator plane, higher tilt of the neutral line for most of the time), the bent (deflected) current sheet structure is partly obscured. The solar equatorial surface is still bent, but the deflection occurs close to the heliopause, inside the layer of mixed polarity. Also, only small parts of the HCS (those with very low tilt) stay close to the bent solar equatorial surface: most of the HCS oscillates between north- and south-deflected plasma flow lines, and is stretched in both directions away from the solar equatorial surface. The expanded fragment of Fig. 5 including a very low tilt HCS segment which indicates the position of the bent solar equatorial surface is shown in Fig. 7.

Figures $8 \mathrm{a}-\mathrm{c}$ show the HCS intersection with the meridional plane for other models of the heliospheric plasma flow: a version of the analytical model of Suess \& Nerney (1990), and two versions of the Bonn model: B1 and B2. The interstellar inflow direction is here assumed to be perpendicular to the solar rotation axis, so that the solar equatorial surface is not bent (we have also considered the case of correctly oriented solar axis and found that the global structure of the HCS was not strongly affected). The time-stationary version of the Bonn model (B1) gives a structure close to the model of Suess and Nerney. The main difference is due to the plasma flow velocity distribution in the heliotail: in all the models presented here except Suess and Nerney the plasma flow slows down near the centre of the heliotail. The time-dependent isotropic solar wind version of the Bonn model (B2) is similar.

Since the Bonn model is axisymmetric, the flow lines are planar. In particular, the lines emerging from the solar poles stay in the meridional plane. Near these flow lines the mixed polarity regions narrow down to a single sheet, separating the unipolar regions of different polarity (compare Fig. 4). For the model B2 with time dependent solar wind (Fig. 8c) these parts of HCS carry also the imprint of interactions between fast and slow streams of the solar wind. Except for the vicinity of the flow
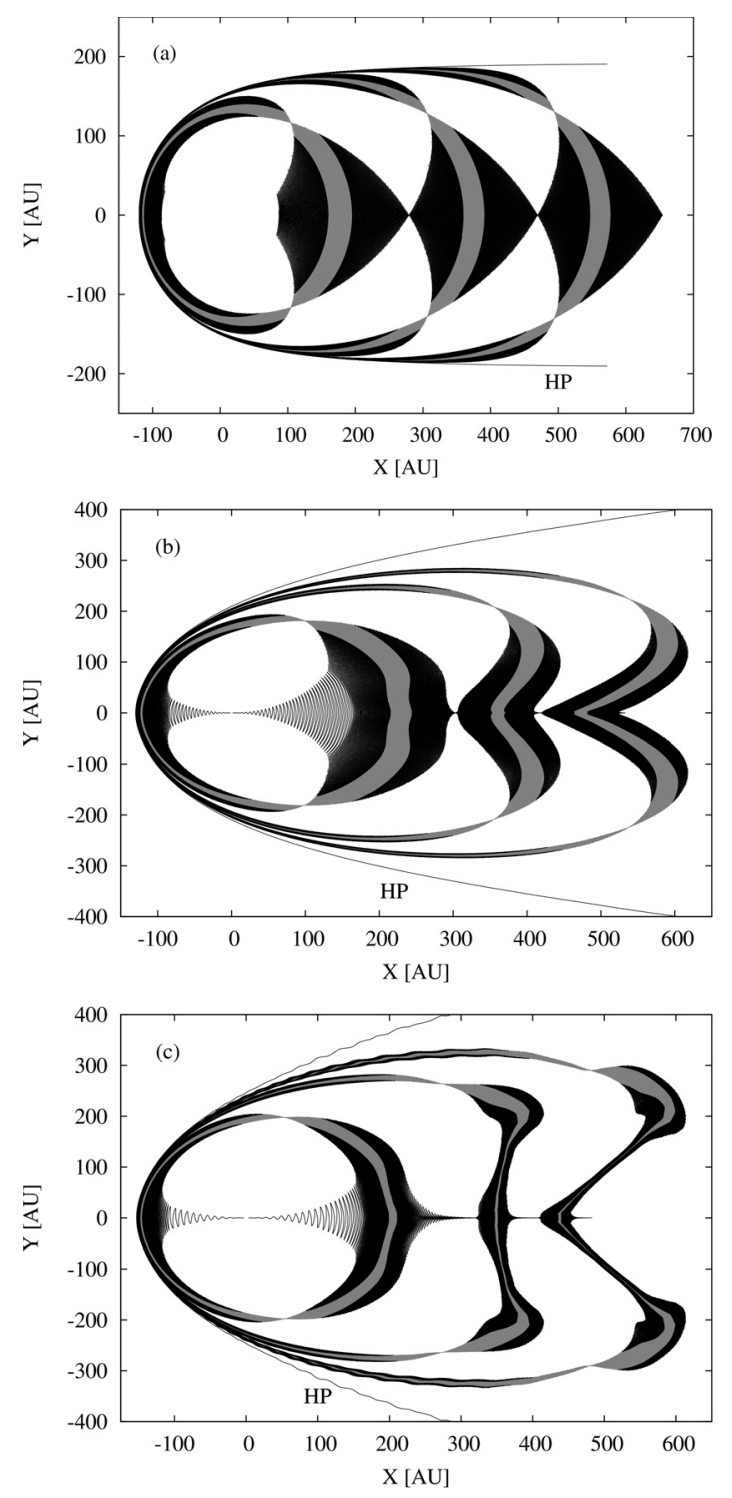

Fig. 8. Intersection of the heliospheric current sheet structure with the meridional plane for different models of the heliosphere at solar minimum: a) a version of the analytical model of Suess and Nerney (the part of the HCS inside the termination shock is not shown), and two versions of the Bonn model: b) B1 and c) B2.

lines emerging from the poles the boundary between the unipolar regions is much thicker (a mixed polarity region including many folds of the HCS).

In Fig. 9 we show a fragment of HCS near the flow line emerging from the south solar pole in the 3D asymmetric model A2. The figure shows the intersection of the HCS with the surface containing the flow line coming out of the pole. Again the boundary between the unipolar regions (white areas) reduces to a single sheet near the polar flow line.

\subsection{Secondary folds}

In Fig. 5 the mixed-polarity regions near the heliopause in the model A2 show secondary folding: that is, the layers filled with the folded HCS bend and fold on themselves. More detailed view is shown in Fig. 10. We find that this secondary folding appears in the cases where the solar wind depends on both latitude and time (A2 but not B2, where the wind is not latitude dependent). 
A. Czechowski et al.: Structure of heliospheric current sheet

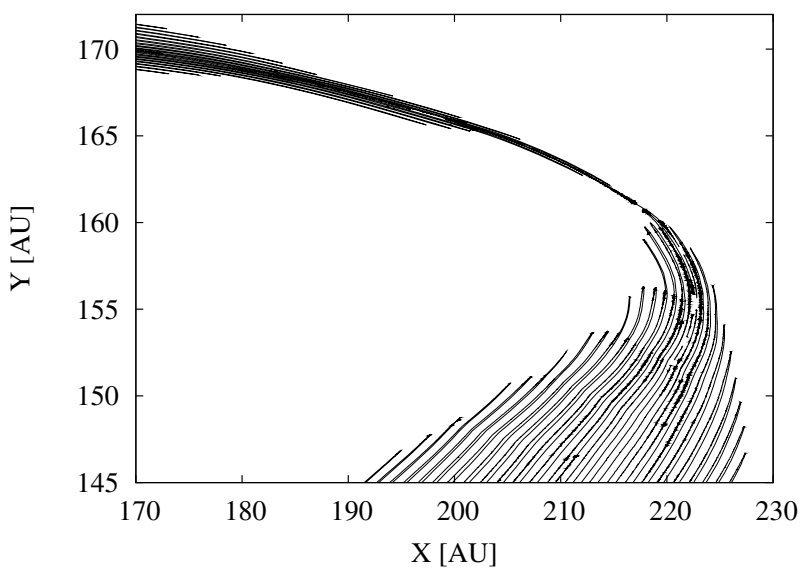

Fig. 9. The HCS structure near the flow line emerging from a solar pole in the MHD model. Along this flow line the unipolar sectors of opposite polarity are separated by a single sheet (compare Fig. 4). Elsewhere, the boundary between the unipolar regions is a mixed polarity region including many folds of the HCS.

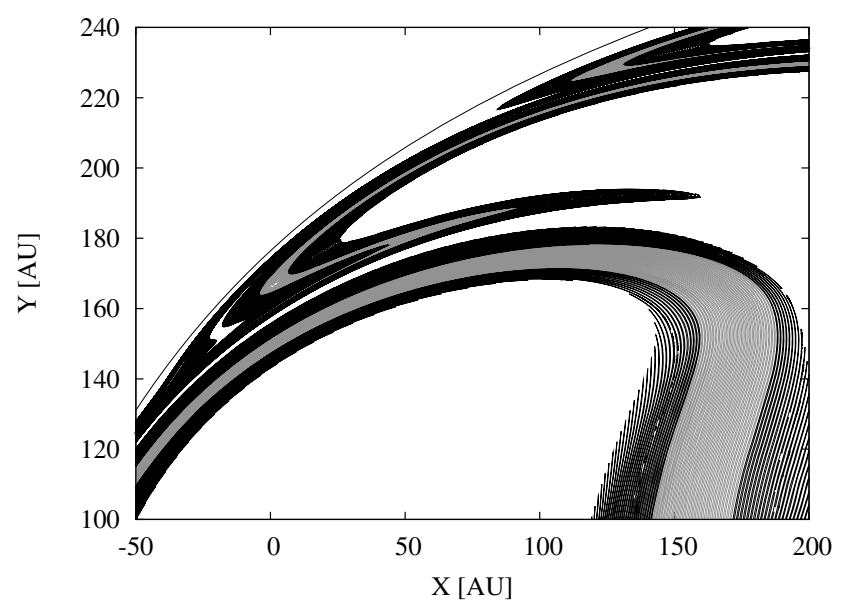

Fig. 10. Detailed view of "secondary fold" structure of the heliospheric current sheet which appears in the model A2 (a part of Fig. 5).

The formation of the fold can be traced back to the evolution of the constant flow time surfaces in the plasma flow. At long distance from the Sun, most of the HCS area become close in shape to these surfaces. This is because, for any pair of points on the section of the HCS situated between the two nearest extremes in heliolatitude, the flow time from the Sun differs by at most $1 / 2$ of the solar rotation time. At long distances from the Sun this is short compared to the overall flow time. Figure 11 presents two constant time surfaces for the model A2. The first is still within the termination shock and reflects the two-component solar wind structure near solar minimum. The second corresponds to a later moment of time, when the flow reached the heliosheath: the boundary between the fast and slow sectors evolves into the fold that corresponds to the secondary fold in the HCS.

In the inner heliosphere, the deformations of the HCS due to variations of the plasma velocity field were considered by Suess \& Hildner (1985) and Pizzo (1994).

\subsection{HCS and mixed polarity sectors near the heliopause}

Convection with the plasma flow moves any given fragment of the HCS from the inner heliosphere, where the spacing between the HCS folds is large, to the heliosheath, where the spacing
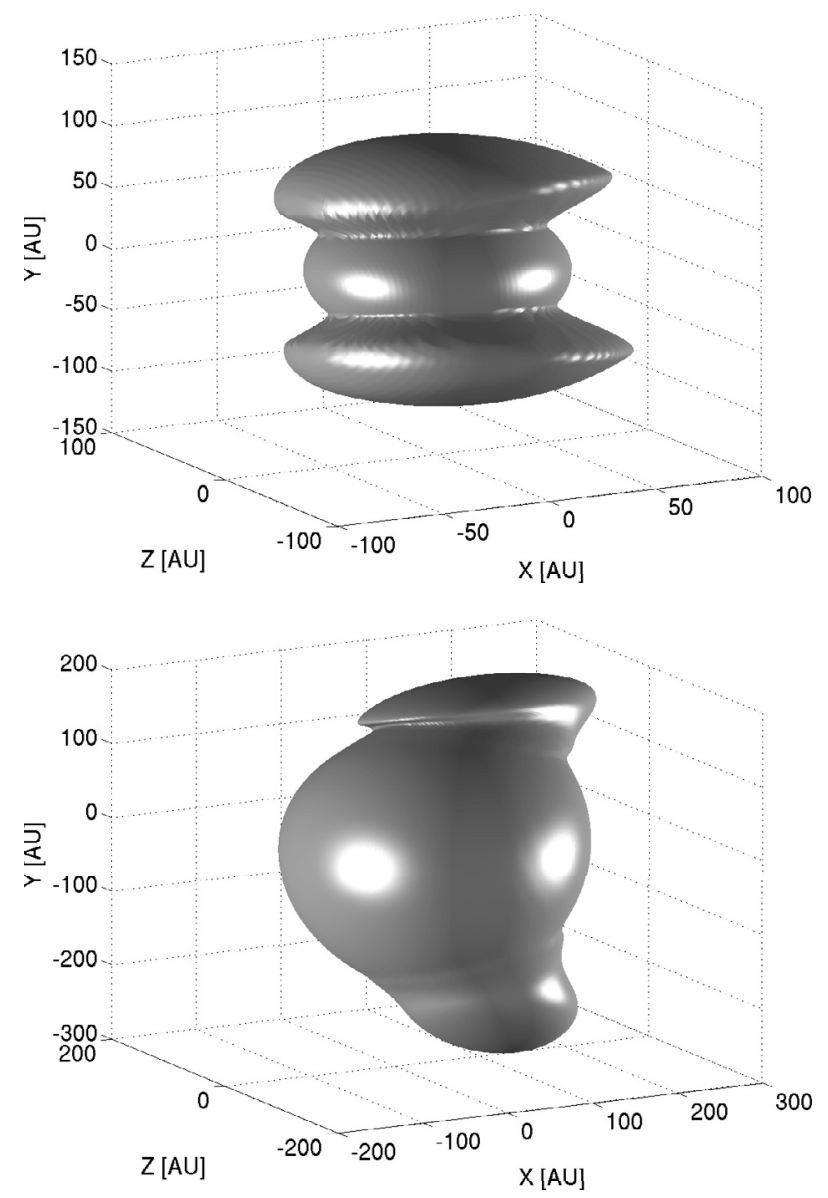

Fig. 11. Constant-time surface for short (at the top) and long (at the bottom) flow-time for the time-dependent MHD model (A2).

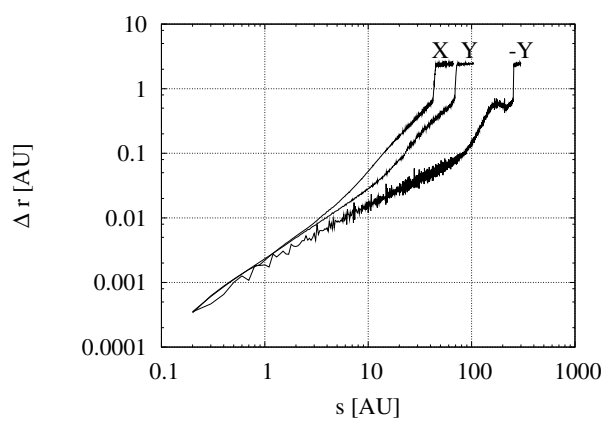

Fig. 12. Distance $\Delta r$ between folds of the heliospheric current sheet as a function of distance $s$ from the heliopause along the following directions: a) interstellar upwind $(X)$, b) crosswind, south $(Y)$, c) crosswind, north $(-Y)$.

decreases and the HCS is stretched by the diverging flow. The freezing-in constraint forbids alternating mixed polarity and the unipolar regions to cross each other, implying that the magnetic polarity regions from previous solar cycles pile up at the heliopause.

As noted before, this pile-up leads to the ultimately nonrealistic compression of the magnetic polarity layers and of the folds in the HCS. We do not know how the situation is resolved. Some speculations are presented in Sect. 4.3.

Figure 12 shows the distance between the subsequent folds of the HCS as a function of the distance from the heliopause. The plots are for three different radial directions in the $\left(\boldsymbol{B}_{\infty}, \boldsymbol{V}_{\infty}\right)$ plane: the interstellar upwind (marked $X$ : towards the "nose" 


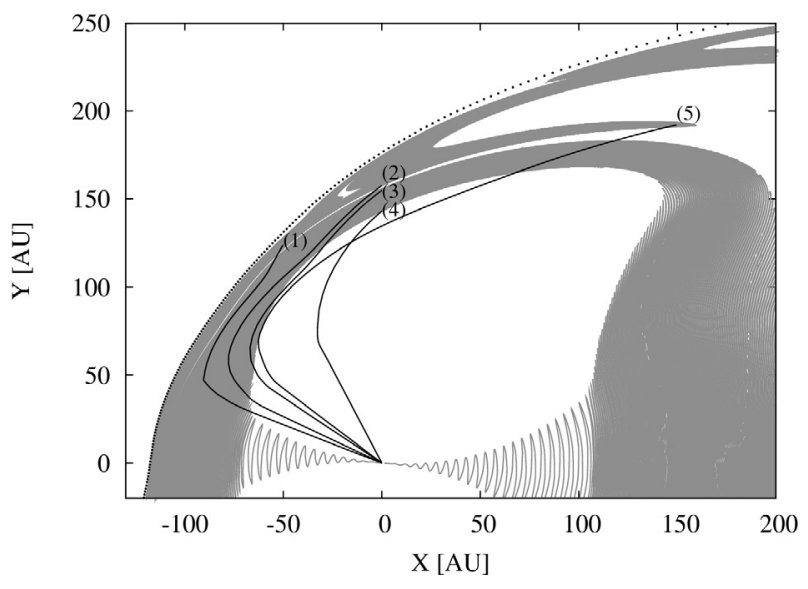

Fig. 13. View of the HCS (model A2, solar minimum) with the plasma flow lines (projected on the $\left(\boldsymbol{B}_{\infty}, \boldsymbol{V}_{\infty}\right)$ plane) terminating in 5 selected points on the HCS. The trajectory reaching the points (4) and (5) avoids the vicinity of the stagnation point.

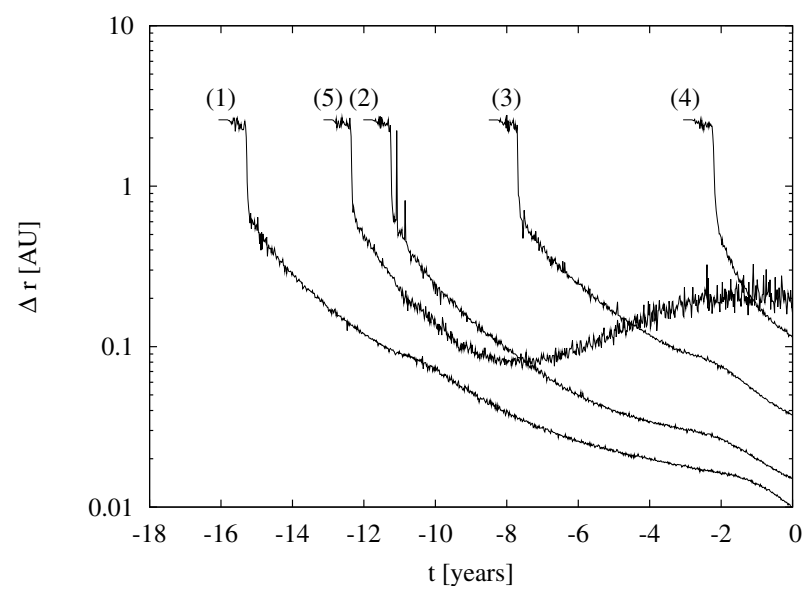

Fig. 14. Estimated distance $\Delta r$ between the subsequent folds in the HCS as a function of time along the flow lines shown in Fig. 13.

of the heliosphere), cross-wind towards south $(+Y)$, and crosswind towards north $(-Y)$. It can be seen that, near the heliopause (within $\sim 5 \mathrm{AU}$ for the forward direction), the spacing between the folds decreases approximately linearly with the distance from the heliopause. This can be understood as a consequence of a similar (approximately linear) decrease with distance from the heliopause in the flow velocity component $v_{\perp}$ perpendicular to the heliopause. Let the linear formula $v_{\perp}(s)=v_{\perp}(L) s / L$ hold for $s \leq L$ where $s$ is the distance from the heliopause. The spacing $\Delta r$ between the folds is then approximately given by

$\Delta r / s=\left[\exp \left(2\left|v_{\perp}(L) / L\right| t_{\mathrm{rot}}\right)-1\right]$

where $t_{\text {rot }}$ is the period of solar rotation. Taking $v_{\perp}(L)=$ $10 \mathrm{~km} \mathrm{~s}^{1}, L=10 \mathrm{AU}$ and $t_{\text {rot }} \sim 2 \times 10^{6} \mathrm{~s}$ it follows that $\Delta r / s \sim 0.01$. This agrees with our results showing that the spacing between the folds in the HCS can be as low as $0.01 \mathrm{AU}$ at the distance of the order of few AU from the heliopause.

The probability of survival of a fragment of the HCS as it approaches the pile-up region may depend on its history. Figure 13 shows examples of plasma flow lines (projected on the $\left(\boldsymbol{B}_{\infty}, \boldsymbol{V}_{\infty}\right)$ plane) terminating in selected points on the HCS. Figure 14 plots the estimated distance $\Delta r$ between the subsequent folds in the HCS along these flow lines. A low value of $\Delta r$ indicates that the folds of the HCS are tightly compressed. We see that the trejectories terminating in the points (5) and (4) do not pass through the region of very low $\Delta r$. If the probability of destruction of the HCS increases for tightly folded HCS, then the points farther away from the heliopause: (5) and (4) are less likely to be affected than the other points. Note that the part of the HCS that avoids the high compression region includes a part (point (5)) of the secondary fold structure.

\section{Discussion}

\subsection{Interstellar magnetic field and the structure of the heliosphere}

In the approximate model of the Sun with spherically symmetric solar wind outflow and with the magnetic fields neglected the heliosphere would be axisymmetric, with the symmetry axis along the direction of motion of the Sun relative to the local interstellar cloud. The effect of the interstellar magnetic field, as derived from theory and numerical simulations (for a review see Zank 1999), is to cause the deformation of the heliosphere which affects both the shape of the outer boundary and the structure of the internal flow: in particular, the shape of the termination shock is also changed. Until recently, there were no observations permitting determination of the interstellar magnetic field in the local interstellar medium.

Voyager observations in the heliosheath and the most recent data from IBEX provided new information about the structure of the distant regions of the heliosphere and the parameters of the interstellar magnetic field. The boundary conditions for the MHD model used in our study are close to the values implied by those recent observations.

We assume the interstellar magnetic field of relatively high strength $3.8 \mu \mathrm{G}$ and direction towards ecliptic (longitude, latitude $)=\left(68^{\circ},-35^{\circ}\right)$ or Galactic $\left(210^{\circ},-33^{\circ}\right)$ which corresponds to the angle $30^{\circ}$ relative to the interstellar helium inflow direction and $20^{\circ}$ relative to the hydrogen deflection plane (the plane defined by the interstellar neutral helium and interstellar neutral hydrogen velocities).

The interstellar magnetic field affects the shape of the heliopause and of the termination shock. In particular, the difference in distance to the termination shock measured by Voyager 1 (94 AU) and Voyager 2 (84 AU) is in part attributed to the interstellar magnetic field. The model of the interstellar field used in our calculations was found to be able to explain this difference in the framework of the stationary model of the heliosphere (Ratkiewicz \& Grygorczuk 2008). It is also close to the results of Opher et al. (2009) (3.7-5.5 $\mu \mathrm{G}$ field strength, angle $20^{\circ}-30^{\circ}$ relative to interstellar inflow and about $30^{\circ}$ from the Galactic plane).

The recent energetic neutral atoms observations by IBEX found a ribbon structure of enhanced emission, thought to be caused by the interstellar magnetic field and reflecting its orientation. Heerikhuisen et al. (2010) reproduce the ribbon structure seen in the IBEX data using the field (parallel to the hydrogen deflection plane) of strength $3 \mu \mathrm{G}$ directed towards $\left(224^{\circ}, 41^{\circ}\right)$ which is (apart from the overall sign change) within about $20^{\circ}$ from our direction. The difference in sign of the interstellar field should not change its effect on the heliosphere, unless the effects of reconnection between the solar and interstellar magnetic fields would be important.

The interstellar magnetic field used in our MHD models is therefore close to the presently favoured values. The field strength and orientation following from the observations are such that one expects a large effect on the structure of the distant heliosphere and in particular on the shape of the heliopause. The 
strongly asymmetric heliosheath used as a basis for our study may be consequently a reasonable qualitative approximation to reality.

One must, however, keep in mind that the present numerical models of the heliosphere are still very incomplete and even the best available boundary conditions may not guarantee a correct description of the plasma flow in the heliosheath. In particular, the instability effects (Dasgupta et al. 2006) may be important near the heliopause.

\subsection{Relation to the numerical models of the heliospheric current sheet}

Numerical models of the heliosphere that include the HCS encounter two challenges. One is that the HCS is very thin. The other is the need to deal with the consequences of the magnetic field reversals.

Magnetic field reversals lead to alternating polarity regions. The heliospheric plasma flow causes a pile-up of these structures near the heliopause. To describe this process requires not only a very high resolution. The model must be also time dependent and the calculation must run over the period of time long enough to include more than one solar cycle. Finally, the model should include the necessary physics to resolve the problems related to the pile-up: for instance, ideal MHD is not sufficient to describe reconnection.

To our knowledge, the most complete numerical calculation up to now is the one by Pogorelov and Borovikov (unpublished) which includes tilted current sheet, has the resolution of $0.01 \mathrm{AU}$ and follows the HCS evolution to the region beyond the termination shock. However, it does not reach the vicinity of the heliopause. The structure of the HCS emerging from this calculation is similar to the results presented here, with the HCS convected by the plasma flow and the HCS folds tightening beyond the termination shock.

The published models are much more simplified (Linde et al. 1998; Washimi \& Tanaka 2001; Opher et al. 2003, 2006; Pogorelov 2006; Pogorelov et al. 2004, 2006, 2009). In particular, the effect of the magnetic field reversals on the HCS is not included. Pogorelov et al. (2009) consider the field reversals but the HCS is not resolved.

The simplified models are a necessary stage in development of more complete ones, but can be inadequate for concrete applications.

We give two examples:

(1) solar magnetic field effect on charged particles entering the heliosphere. Since the simplified models ignore the sector structure of the magnetic field, the reduction of the effective magnetic field leading to the increased probability of particle penetration into the inner solar system (Landgraf 2000; Czechowski \& Mann 2003) is missing from these models;

(2) bending of the HCS. In calculations with low tilt heliospheric current sheet, the current sheet was found to avoid the stagnation point by bending into the northern or the southern parts of the heliosheath, with the shape of the heliopause becoming asymmetric in result (Pogorelov et al. 2004). If the solar cycle would be taken into account, the HCS would extend to all latitudes and so could not avoid the stagnation point in this way. The effect on the shape of the heliopause is then in doubt.

Numerical MHD models of the heliosphere that include the heliospheric magnetic field and the current sheet have clearly an
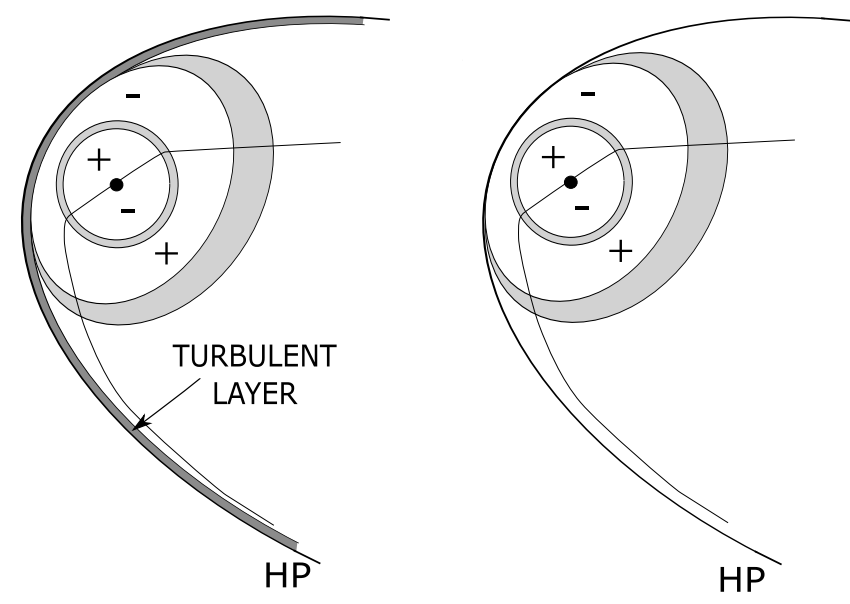

Fig. 15. Schematic view of two scenarios for the magnetic field structure in the forward heliosheath. In the figure on the left the layers of tightly folded HCS alternating with unipolar regions become replaced near the heliopause by a turbulent region of disordered magnetic field. In the figure on the right a unipolar region reaches the heliopause in result of destroying the previous magnetic field structure by reconnection. The grey rings represent the mixed polarity regions and the thin line the bent solar equatorial surface. HP marks the heliopause.

advantage over the non self-consistent approaches like the one used in this paper. However, the effects due to non-realistic model assumptions or numerical effects must be understood and eliminated. In the meantime, the numerical simulations need to be supplemented by other methods.

\subsection{Pile-up region in the forward heliosheath}

As noted in the Introduction, we assume that in the immediate vicinity of the heliopause (within distance $\Delta$ ) magnetic field must be rearranged to avoid unphysical compression of the polarity sectors or the HCS. Here we briefly discuss two of the possible results of this rearrangement, illustrated in Fig. 15.

One possibility is that the mixed polarity field would become disordered. In the region near the heliopause there are reasons to expect increased turbulence (Fahr et al. 1986; Borovikov et al. 2008), in particular in the plasma velocity field. This may combine with magnetic reconnection and lead to the HCS and the magnetic field structure with less long-range order. It is possible that unrealistic compression could then be avoided. The overall picture would still be similar to that of Nerney, Suess and Schmahl, with the mixed polarity regions near the heliopause filled by the disordered field and fragmented current sheets rather than the regularly alternating field and continuous HCS.

Another possibility is that, near the nose of the heliopause, the unipolar regions would extend to the heliopause. This could occur as follows. Assume that at the heliopause the field is unipolar, with negative polarity. It is approached by the expanding positive polarity region from the following solar minimum. Between the unipolar regions there is a mixed-polarity layer. A "hole" in this layer may be created by reconnection between the alternating fields within the layer. The negative polarity field near the heliopause may then reconnect with the positive polarity field in the newly arriving unipolar region, so that the "hole" extends to the heliopause. The edges of the "hole" are convected away from the "nose" by the diverging plasma flow, while the "hole" fills up by the newly arrived plasma with positive polarity field. 
The magnetic field would then form a unipolar layer next to the nose of the heliopause, with the polarity changing over the 11 year solar cycle. Farther away from the nose the structure of the field would be the same as that of Nerney et al. (1991, 1993, 1995).

This scenario requires that very near the heliopause there is still a clear difference between unipolar and mixed polarity sectors and that the reconnection is effective enough to destroy the unipolar layer adjacent to the heliopause. The reason we mention this possibility is that it is similar to the results of simplified numerical models (see Sect. 4.2) which, however, do not include the polarity reversal effects.

\section{Conclusions}

We followed the pioneering work by Nerney et al. (1991, 1993, 1995) by extending their calculations of global magnetic field structure in the heliosphere to more elaborate models of the heliospheric plasma flow. We assumed that the magnetic field is frozen into the plasma and that the HCS is a tangential discontinuity, passively convected with the plasma flow.

The global HCS structure following from the highly asymmetric and time-dependent model of the heliosphere differs in some respects from that obtained by Nerney, Suess and Schmahl in a much simpler (axisymmetric, time-stationary and incompressible flow) model. It is similar in that the magnetic field in the heliosphere forms expanding unipolar regions separated by shells of mixed polarity, and that there is a mixed polarity layer near the heliopause. The differences include: the HCS secondary folding caused by the time- and latitude dependent solar wind; bending of the solar equatorial surface due to asymmetry in the stagnation point position; effect of the nonuniform flow in the heliotail on the shape of the different polarity regions.

Near the heliopause the alternating layers of different polarity become increasingly compressed by the slowing plasma flow. On approaching the heliopause this compression becomes unrealistically high. This is a general consequence of combining the ideal MHD equations for the heliosphere with the solar cycle variation of the magnetic field, including the polarity reversals. To find out how the problem is resolved requires a more detailed study of the physical processes near the heliopause and of the physics of the HCS.

Numerical simulations of the HCS are necessary to understand the HCS structure. These simulations are very difficult because of the need for a very high resolution. The results available at present are based on simplified assumptions and are not adequate for some applications. Our approach, which combines the results of the flow simulations with a description of the HCS based on simple assumptions about its dynamics, has an advantage of being able to model the situations difficult to describe in a self-consistent simulation. Such models may be useful for a study of the charged particles dynamics in the inner heliosheath.

Acknowledgements. We acknowledge support from the Polish Ministry of Science and Higher Education grant 4T12E 002 30. R.R. was supported by the Polish Ministry of Science and Higher Education grant N N203 415933.

\section{References}

Balogh, A., \& Smith, E. J. 2001, Space Sci. Rev., 97, 147

Borovikov, S. N., Pogorelov, N. V., Zank, G. P., \& Kryukov, I. A. 2008, ApJ, 682,1404

Burlaga, L. F., Ness, N. F., \& Richardson, J. D. 2003, J. Geophys. Res., 108, 8028
Burlaga, L. F., Ness, N. F., \& Acuna, M. H. 2006, in Physics of the Inner Heliosheath: Voyager Observations, Theory and Future Prospects, 5th IGPP International Astrophysics Conference, ed. J. Heerikhuisen, V. Florinski, G. P. Zank, \& N. V. Pogorelov, AIP Conf. Proc., 858, 122

Burlaga, L. F., Ness, N. F., \& Acuña, M. H. 2007, ApJ, 668, 1246 Burlaga, L. F., Ness, N. F., Acuña, M. H., et al. 2009, ApJ, 692, 1125 Czechowski, A., \& Mann, I. 2003, J. Geophys. Res., 108, A10, LIS 13-1 Dasgupta, B., Florinski, V., Heerikhuisen, J., \& Zank, G. P. 2006, in Physics of the Inner Heliosheath: Voyager Observations, Theory and Future Prospects, 5th IGPP International Astrophysics Conference, ed. J. Heerikhuisen, V. Florinski, G. P. Zank, \& N. V. Pogorelov, AIP Conf. Proc., 858, 51

Decker, R. B., Krimigis, S. M., Roelof, E. C., et al. 2005, Science, 309, 2020 Decker, R. B., Krimigis, S. M., Roelof, E. C., et al. 2008, Nature, 454, 67 Fahr, H. J., Kausch, T., \& Scherer, K. 2000, A\&A, 357, 268

Fahr, H. J., Neutsch, W., Grzedzielski, S., Macek, W., \& Ratkiewicz-Landowska, R. 1986, Space Sci. Rev., 43, 329

Gosling, J. T., McComas, D. J., Skoug, R. M., \& Smith, C. W. 2006, GRL, 33, L17102

Heerikhuisen, J., Pogorelov, N. V., Zank, G. P., et al. 2010, ApJ, 708, L126

Jokipii, J. R., \& Levy, E. H. 1977, ApJ, 213, L85

Landgraf, M. 2000, J. Geophys. Res., 105, A5, 10303

Linde, T. J., Gombosi, T. I., Roe, P. L., Powell, K. G., \& DeZeeuw, D. L. 1998, J. Geophys. Res., 103, A2, 1889

Miyake, S., \& Yanagita, S. 2005, in Proceedings of 29th International Cosmic Ray Conference, Pune, 101

Nerney, S., Suess, S. T., \& Schmahl, E. J. 1995, J. Geophys. Res., 100, A3, 3463 Nerney, S., Suess, S. T., \& Schmahl, E. J. 1993, J. Geophys. Res., 98, A9, 15169 Nerney, S., Suess, S. T., \& Schmahl, E. J. 1991, A\&A, 250, 556

Opher, M., Liewer, P. C., Gombosi, T. I., et al. 2003, ApJ, 591, L61 Opher, M., Stone, E. C., \& Liewer, P. C. 2006, ApJ, 640, L71

Opher, M., Alouani Bibi, F., Toth, G., et al. 2009, Nature, 462, 1036 Pizzo, V. J. 1994, J. Geoph. Res., 99, A3, 4185

Pogorelov, N. V., Borovikov, S. N., Zank, G. P., \& Ogino, T. 2009, ApJ, 696, 1478

Pogorelov, N. V. 2006, in Physics of the Inner Heliosheath: Voyager Observations, Theory and Future Prospects, 5th IGPP International Astrophysics Conference, ed. J. Heerikhuisen, V. Florinski, G. P. Zank, \& N. V. Pogorelov, AIP Conf. Proc., 858, 3

Pogorelov, N. V., Zank, G. P., \& Ogino, T. 2004, ApJ, 614, 1007

Pogorelov, N. V., Zank, G. P., \& Ogino, T. 2006, ApJ, 644, 1299

Pudovkin, M. I., \& Semenov, V. S. 1977a, Ann. Geophys., 33, 429

Pudovkin, M. I., \& Semenov, V. S. 1977b, Ann. Geophys., 33, 423

Ratkiewicz, R., \& Grygorczuk, J. 2008, Geophys. Res. Lett., 35, No. 23, CiteID L23105

Ratkiewicz, R., Barnes, A., Molvik, G. A., et al. 1998, A\&A, 335, 363

Ratkiewicz, R., Ben-Jaffel, L., \& Grygorczuk, J. 2008, Astron. Soc. Pac. Conf. Ser., 385, 189

Richardson, J. D., Stone, E. C., Kasper, J. C., Belcher, J. W., \& Decker, J. B. 2009, GRL, 36, L10102

Riley, P., Linker, J. A., \& Mikic, Z. 2002, JGR, 107, A7, 1136

Scherer, K., \& Fahr, H. J. 2003a, Geophys. Res. Lett., 30, No. 2, 17-1

Scherer, K., \& Fahr, H. J. 2003b, Annnales Geophys., 21, 1303

Schwadron, N. A., Bzowski, M., Crew, G. B., et al. 2009, Science, 326, 966

Smith, E. J. 2001, J. Geophys. Res., 106, No. A8, 15819

Smith, E. J., Balogh, A., Forsyth, R. J., \& McComas, D. J. 2001, Geophys. Res. Lett., 28, 4159

Smith, E. J. 2008, in The heliosphere through the solar activity cycle, ed. A. Balogh, L. J. Lanzerotti, \& S. T. Suess, Springer Praxis Books (Berlin, Heidelberg: Springer), 79

Stone, E. C., Cummings, A. C., McDonald, F. B., et al. 2005, Science, 309, 2017 Stone, E. C., Cummings, A. C., McDonald, F. B., et al. 2008, Nature, 454, 71

Suess, S. T., \& Hildner, E. 1985, J. Geophys. Res., 90, A10, 9461

Suess, S. T., \& Nerney, S. 1990, J. Geophys. Res., 95, 6403

Suess, S. T., \& Nerney, S. 1993, Geophys. Res. Lett., 20, 329

Usoskin, I. G., Alanko-Huotari, K., Mursula, K., \& Kovaltsov, G. A. 2008, in Proceedings of the 30th International Cosmic Ray Conference, ed. R. Caballero, J. C. D’Olivo, G. Medina-Tanco, L. Nellen, F. A. Sanchez, \& J. F. Valdes-Galicia, Univ. Nacional Autonoma de Mexico, Mexico City, 1, 459 Washimi, H., \& Tanaka, T. 2001, Adv. Space Res., 27, 509

Winterhalter, D., Smith, E. J., Burton, M. E., Murphy, N., \& McComas, D. J. 1994, JGR, 99, 6667

Zank, G. P. 1999, Space Sci. Rev., 89, 413

Zhou, X.-Y., Smith, E. J., \& Winterhalter, D. 2005, in Proceedings of Solar Wind 11-SOHO 16, Connecting Sun and Heliosphere, Whistler, Canada 12-17 June 